\title{
Hydroxychloroquine-induced Hyperpigmentation
}

Amir Bajoghli, MD, Skin \& Laser Surgery Center, McLean, and INOVA Fairfax Hospital, Falls Church, Virginia; Gabriela Hernandez, BS, Skin \& Laser Surgery Center, McLean, Virginia; Michael A. Cardis (1), MD, Medstar Washington Hospital Center/Georgetown University Hospital, Washington, DC, USA. Address correspondence to Dr. M.A. Cardis, 5530 Wisconsin Ave., Suite 730, Chevy Chase, MD 20745, USA. Email: michael.a.cardis@medstar.net. Written consent for publication of the photos herein has been waived because they are nonidentifiable. Institutional review board approval has been waived because this is a case report involving a single patient case that does not meet the US Department of Health and Human Services definition of "research."

About $10-25 \%$ of patients with systemic lupus erythematosus (SLE) managed with antimalarials will develop cutaneous hyperpigmentation, and although the majority of such cases is attributed to chloroquine, hydroxychloroquine (HCQ) is also implicated and is likely underrecognized ${ }^{1}$.

A 52-year-old Hispanic woman with SLE and endstage renal disease receiving peritoneal dialysis presented with a 3-month history of asymptomatic, progressive darkening of her bilateral upper extremities. There was no antecedent trauma or preceding skin eruption. She had been taking HCQ $200 \mathrm{mg}$ daily for 12 years. Physical examination revealed diffuse mottled and reticular hyperpigmentation with adjacent areas of ecchymosis on

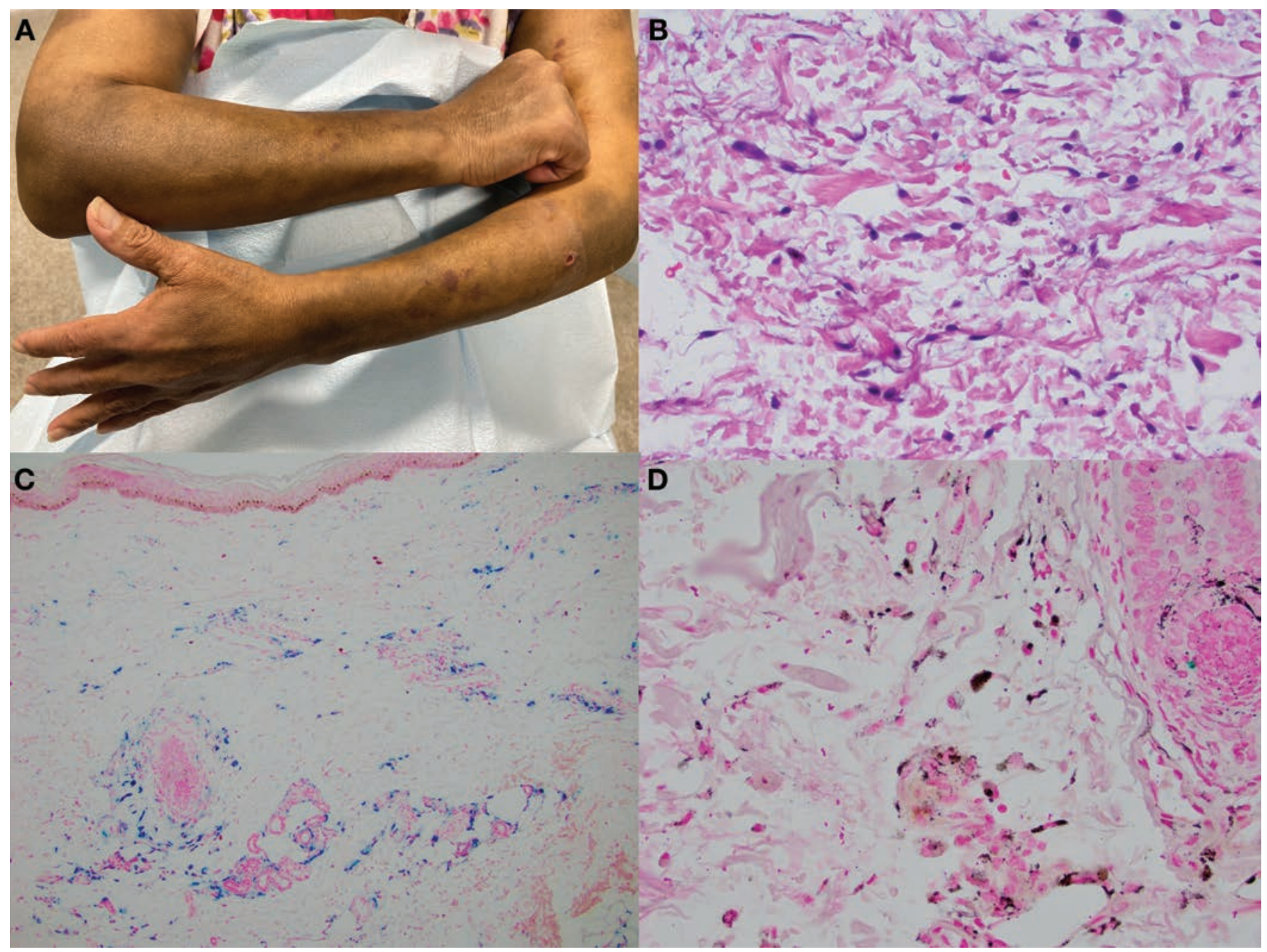

Figure 1. A. Mottled and reticular hyperpigmented patches on the arms with focal ecchymosis. B. High-magnification H\&E-stained section (original magnification $\times 400$ ) showing scattered pigmented granules within the mid dermis, some seen within histiocytes. C-D. Perls' Prussian blue (original magnification $\times 100)$ and Fontana Masson (original magnification $\times 400$ ) stains reveal significant iron and melanin deposits within the dermis, respectively. 
the bilateral upper extremities (Figure 1A). A biopsy revealed dermal deposits of iron and melanin (Figure 1B-D). A diagnosis of drug-induced dyschromia was made with a staining pattern compatible with that of antimalarial exposure.

Presentation on the lower legs, especially shins, is classic, but other sites may be involved including mucosa and nail beds ${ }^{2}$. The pigmentation may begin within a year of treatment, but the timing of onset is variable, and contrary to HCQ-associated retinopathy, a direct association between cumulative dose or duration of therapy with the pigmentation has not been substantiated ${ }^{1}$. The pathophysiology is not well understood, but it is thought that the pigmentation is precipitated by bruising leading to hemosiderin deposition and subsequent activation of melanocytes leading to the accumulation of melanin. This hypothesis, however, is inadequate because it does not account for pigmentation on nontraumatic sites such as mucosa. Upon cessation of HCQ, some patients demonstrate partial improvement, and laser treatments may improve residual dyschromia ${ }^{3}$.

\section{REFERENCES}

1. Bahloul E, Jallouli M, Garbaa S, Marzouk S, Masmoudi A, Turki H, et al. Hydroxychloroquine-induced hyperpigmentation in systemic diseases: prevalence, clinical features and risk factors: a cross-sectional study of 41 cases. Lupus 2017;26:1304-8.

2. Jallouli M, Francès C, Piette JC, Huong DL, Moguelet P, Factor C, et al. Hydroxychloroquine-induced pigmentation in patients with systemic lupus erythematosus. JAMA Dermatol 2013;149:935-40.

3. Coulombe J, Boccara O. Hydroxychloroquine-related skin discoloration. CMAJ 2017;189:E212 